Session 1332

\title{
Three Dimensional Circuit Animation Visualizes Voltage
}

\author{
Charles R Sullivan \\ Thayer School of Engineering, Dartmouth College
}

\section{Introduction}

The fundamental variables in electrical circuits — voltage and current — are not directly visible to students, and must be measured indirectly with meters and oscilloscopes. This is in contrast to, for example, mechanical systems, in which position and velocity are directly visible, and force can be directly felt. (Voltage and current can also be felt, but doing so is unpleasant and usually unsafe.) This lack of visibility impedes learning about circuits in many ways, not only in lab work, but also in communication about circuits in the classroom and in paper or electronic documents, and more generally, because without a way to mentally visualize circuits, it can be more difficult to gain an intuitive understanding of circuit operation.

With modern computer hardware and software tools, animations can be readily produced, and have been used to demonstrate circuit behavior ${ }^{1,2,3,4,5,6,7,8}$. The objectives of these animations vary, from commercial software intended to approximate a lab experience with a photorealistic representation of lab instruments and circuit breadboarding (but with no attempt to visualize anything more than what is visible in a real lab) ${ }^{8}$, to a systematic visualization of all invisible circuit quantities: current, voltage, power flow and energy storage ${ }^{1,2}$. Our objective is to represent the two main variables, voltage and current, as clearly and intuitively as possible.

It is not immediately clear how best to visualize current and voltage. However, among those who have considered it, there is consistent enthusiasm for representing voltage using a threedimensional (3D) circuit diagram ${ }^{1,2,3,7,9}$. The first two dimensions are used for the layout of the circuit, as in a standard schematic, but the third dimension is used to represent voltage, with the vertical position of a wire corresponding to its potential. As discussed in Section II.A, we found that this visualization method, as shown in Fig. 3, best met our objectives. There is less of a consensus on good representations for current. As discussed in Section II.B, we chose to use arrows with the length of the arrow corresponding to current magnitude, as in Jim Hauser's 3D illustrations in White ${ }^{9}$. Using the MATLAB language ${ }^{10}$, we developed a tool kit to generate 3D animated diagrams in this format. The tool kit, freely available via the web ${ }^{11}$, is similar to the Mathematica-based CircuitViz system ${ }^{2}$, but, in addition to being based on a different platform, it uses a different current representation and adds the use of color. These and other details of the visualization are discussed in Section II. Section III describes the MATLAB implementation, and Section IV discusses experiences with using the system in the classroom. Some possible improvements are discussed in Section V. 


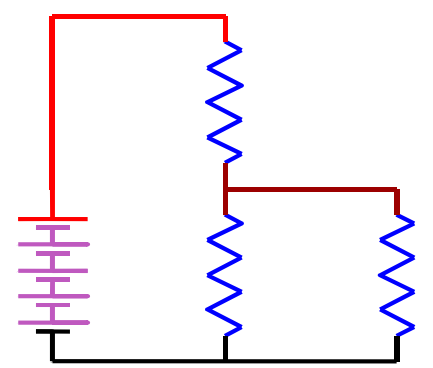

Fig. 1. A dc circuit. If you are viewing this in color, the shading of the nodes represents voltage on a scale from black, corresponding to zero volts, to red, representing $10 \mathrm{~V}$.

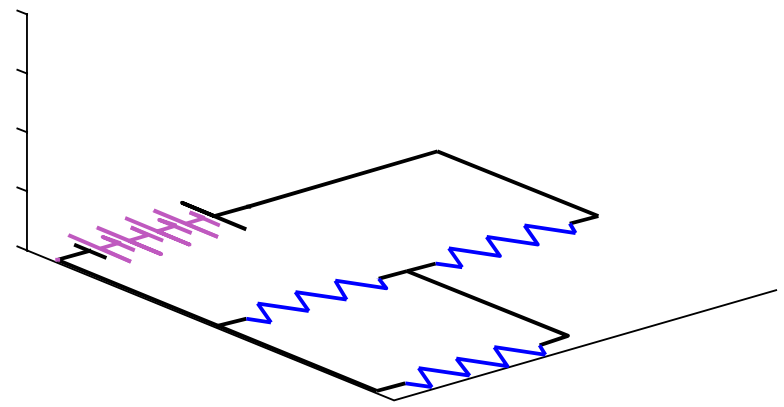

Fig. 2. The same circuit as in Fig. 1, in a 3D perspective view, with the third dimension unused.

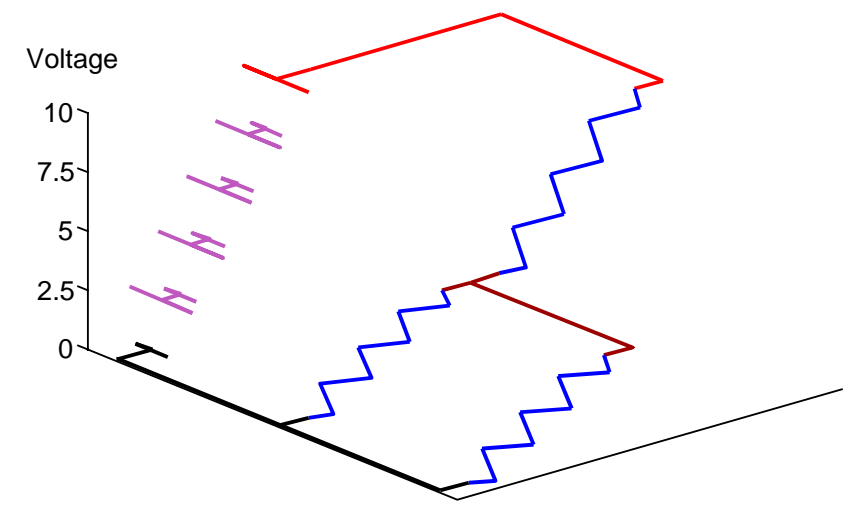

Fig. 3. The same circuit as in Fig. 1 and 2, with the third dimension used to represent voltage.

II. Selecting a visualization system

A. Use of three dimensions for visualization of voltage

An experienced electrical engineer can look at a circuit diagram and immediately "see" that the voltage differences across two parallel elements are the same, whereas a beginning student may need to define variables, write an explicit Kirchoff's Voltage Law (KVL) equation, and often then correct the sign errors made in these steps in order to arrive at the same conclusion.

Helping students gain intuition for KVL is important to helping them get to a higher level of

Proceedings of the 2002 American Society for Engineering Education Annual Conference \& Exposition Copyright (C) 2002, American Society for Engineering Education 
proficiency more quickly. We would like for them to be able to literally see how voltage drops across different elements are related. Understanding Kirchoff's Current Law (KCL) is also important, although it seems easier for students to grasp this intuitively, based on the idea of conservation of charge. Thus, illustrating KCL is also an important objective, but a good representation of voltage takes priority.

As discussed in more detail by Doering ${ }^{2}$ and the references cited therein, it is valuable to make the display of variables integral to the representation of the system-meaning, in this case, on the circuit diagram, and, to the extent possible, integrated with the symbols representing components and connections on the diagram. An adjacent graph or other indicator can be useful, but the process of matching of legends, colors, or other indications is distracting, and is always one step removed from the circuit. Our goal is to enable students to see what is happening by watching the circuit without any extra mental steps.

One possible way of representing voltages on a circuit diagram include indicators such as bar graphs, meter analogs, or even text numbers next to components or nodes ${ }^{5,6}$. However, it is hard to see how such a representation could be visually related to KVL. One way to visually demonstrate sums of voltages would be to use a stacked bar graph, with the sum of the individual bars equaling the total height of the stack. This idea could be extended to deal with negative numbers through the approach shown in Fig. 4. However, such a diagram removes the voltage indication from the nodes or components in order to place all the voltages together. It also requires the extra step of polarity definitions on the circuit diagram. Thus, it only fulfils a few of our objectives for a voltage representation.

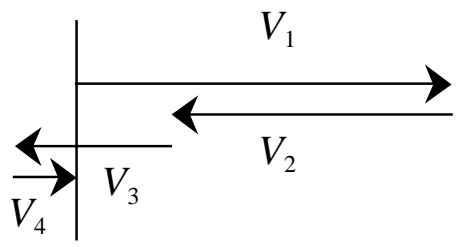

Fig. 4. A voltage vector representation that could be used to illustrate KVL, but that necessarily separates the voltage indication from the circuit element.

A well known and very useful way to indicate voltages on a circuit diagram is to shade nodes in different colors corresponding to voltage, as in Fig. 1. It can be effective on the blackboard, and is also something students can do on their own as they develop equations to model circuits. It is particularly effective in demonstrating which components are in parallel, and in demonstrating that those components therefore must all have the same voltage drop, but it is less effective at demonstrating the magnitudes of the voltages involved. A scale might, for example, indicate that a voltage drop from green-to-orange is about twice the voltage of orange-to-red, but it is hard to "see" that directly from the colors on the circuit diagram. 
Compared to the alternatives, the advantages of using the third dimension to represent voltage are substantial. The representation is not just adjacent to the elements of the diagram, but is fully integrated into the diagram. Although the perspective drawing does not make it easy to precisely match node elevations to the voltage scale on the left, it does take advantage of humans' innate ability to make semi-quantitative judgments given such a view ${ }^{2}$. For example, it is easy to see in Fig. 3 that the voltage across the two parallel resistors is smaller than the voltage across the other resistor. This representation also has the advantage of providing an intuitive analogy between gravitational potential energy and electrical potential energy, although, as noted in Doering ${ }^{2}$, one must be careful to explain the limitations of this analogy.

However, the biggest advantage of the representation in Fig. 3 is that it makes KVL immediately apparent, and in fact makes the very idea of violating it as counter-intuitive as an M.C. Escher drawing. One can easily see not only that the voltage drop across the two parallel resistors must be equal, but also that the sum of the drop across the parallel combination plus the voltage drop across the other resistor must equal the voltage of the source.

Thus, the 3D representation with elevation corresponding to voltage meets our objectives very effectively, and we have chosen to use it for the animation system we have developed. We also shade nodes according to voltage. This is intended to communicate the same voltage information over more channels, to correspond to what is shown in the textbook and on the blackboard, and to reinforce the suggestion that students color nodes themselves as they analyze circuits.

For resistors and inductors, the voltage is shown dropping linearly over the length of the active element. However, for capacitors, the potential of the plate should be the same as the potential of the wire it is connected to, and this is how we depict it (see, for example, Fig. 5). This follows Elec3 $\mathrm{D}^{3}$, but differs from the representation used in CircuitViz ${ }^{2}$, where a section of the diagram including the capacitor and its leads is "ramped" the same as resistors. The approach of directly representing capacitor plate potential has advantages and disadvantages. The main disadvantage is that for capacitors charged to a large potential, it can look like the two widely separated plates are separate, unrelated elements; it can be hard to see that they are in fact the two plates of one

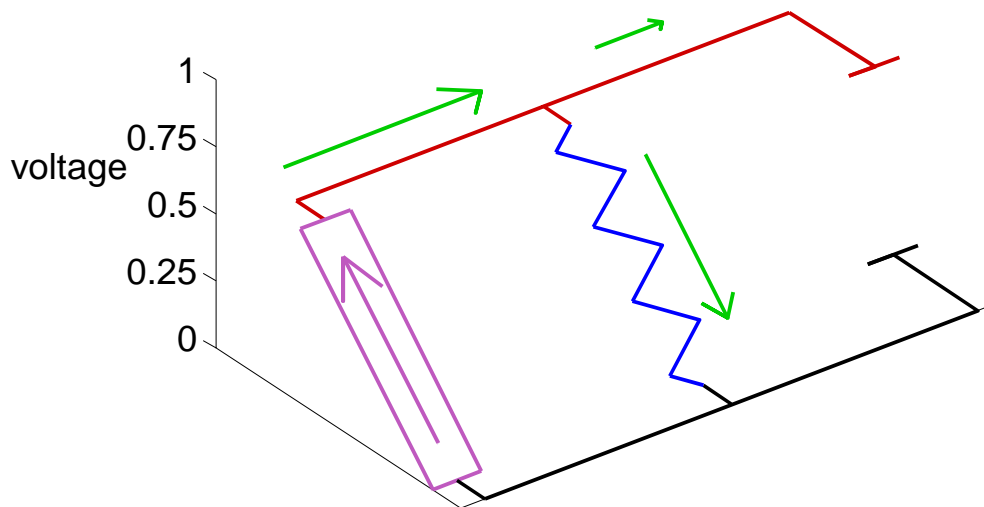

Fig. 5. A snapshot of an animation of a parallel RC circuit charging from a current source. 
capacitor. However, this representation more directly depicts what is going on physically, and we also found that it had an additional benefit: It makes the appearance of capacitors significantly different from that of resistors, providing a visual correlate to their widely different behavior. It also allows us to consistently keep all wires (including component leads) horizontal, reinforcing that idea that voltage drop along a wire is always zero.

We used a new way to represent voltage on a diode that is a hybrid between the two styles, as shown in Fig. 6. When the diode is off, the two ends of the diode separate, and the potentials each follow their respective nodes. However, when the diode is conducting, the body of the diode is shown slanted, indicating a voltage drop across the junction, without the disconnection effect shown in the reverse-bias case.

\section{B. Visualization of current}

Although we have four precedents for the use of the third dimension to represent voltage $e^{2,3,7,9}$, they all use different representations of current, or omit current from the visualization on the schematic $^{3}$. Because current is motion of charge, moving objects can represent that motion, for example with small spheres of charge moving along a circuit path ${ }^{7}$ or with dots moving along wires in a schematic ${ }^{4,5}$. Either the speed or the density of moving carriers could be varied to represent the magnitude of current. At least in a wire, variations in velocity are better representative of the physical behavior than are changes in carrier density, with the caveat that the velocity shown represents only the net drift velocity and not the thermal motion (adding random thermal motion would not help the clarity of the animation). However, this has a limitation similar to that of using color for voltage: quantitative relationships are difficult to judge.

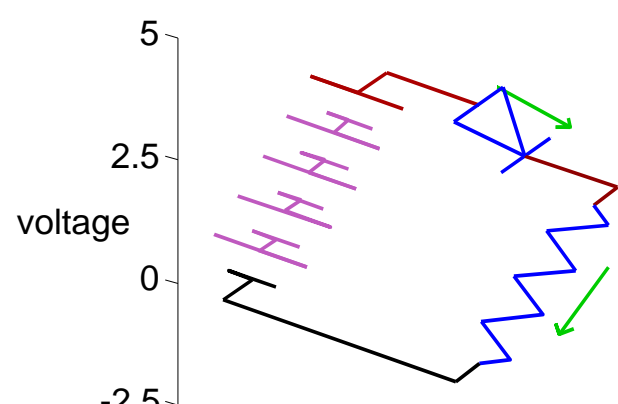

$-2.5$

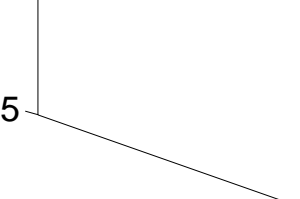

Fig. 6. Two snapshots of an animation of a simple diode rectifier. When the diode is on, the voltage drop is shown occurring across the arrow portion of the diode. When the diode is off, it is shown disconnected. The logical state can be controlled independent of the voltage polarity, in order to illustrate reverse-recovery, if desired. 
In CircuitViz ${ }^{2}$, current is represented by a vertical ribbon underneath of the current path, with the vertical width of the ribbon representing current magnitude, and arrows on the top indicating current direction. This is an excellent choice for several reasons. Firstly, it has some basis in a physical analogy to water flow, with a deeper channel having greater flow. Secondly, it locates the indication coincident with the current path on the schematic, and in doing so highlights the main current flow path in the circuit. Finally, it allows qualitative judgments of magnitude, as does the vertical representation of voltage. For example, on can see that KCL holds at a node by seeing that the ribbon heights beneath two paths sum to the ribbon height of the third.

However, this representation also has disadvantages. First, voltage and current are both indicated as vertical variations. Thus, more careful attention is needed to focus on one or the other, or, more importantly, to gain intuition about their relationship in a component. One must know that the top edge of the ribbon, not the bottom edge, is indicating voltage. Second, the indication does not integrate the representation of the direction of the current as fully or naturally as the use of elevation does with voltages. Separate arrows on the wires are used, but they can be hard to see, and they require attention to yet another aspect of the image.

There does not appear to be any one best way to represent current, but we chose to use the method in the 3D illustrations in White ${ }^{9}$, which is simply an arrow adjacent to a component or branch with the length of the arrow representing magnitude, as in Fig. 5. Although a violation of KCL could easily be rendered without Escher's assistance, it is possible to see in Fig. 5 that the lengths of the two arrows for the passive components approximately sum to the length of the arrow for the current source. The length of the arrow and the position of its head are in some sense different indications for direction and magnitude — something we wish to avoid — but we find that they are closely enough linked, perhaps because the arrow is a very familiar symbol, that the result is readily interpreted as a single indicator showing direction and magnitude.

One objective that this satisfies is that the representations of voltage and current should be as different as possible, since these are fundamentally different quantities. The direction in which the current arrow grows and shrinks is generally orthogonal to the vertical direction of voltage. At the same time, however, the arrows tilt to follow the potential difference between two nodes. This means that current arrows for resistors always point downhill, and that current arrows for inductors always grow in the downhill direction or shrink in the uphill direction. This builds intuition both by showing a consistent correlation, and by drawing on the gravitational analogy.

Two disadvantages of the arrow representation for current are apparent, however: Generally, putting arrows on each branch or wire leads to a cluttered diagram, so it is wise to place the arrows only in key places. However, this relies on the viewer to figure out where the full current path is by following wires or other branches beyond the explicit arrows. This is a significant disadvantage compared to CircuitViz ${ }^{2}$ where primary current paths are highlighted by the thicker ribbon underneath of them. Also, it is important for students to understand that the meaning of the arrow is different from the meaning of an arrow used when defining a current variable: The animation arrows indicate the actual current direction, whereas the definitional arrows indicate 
only which direction is called positive. CircuitViz ${ }^{2}$ includes both types of arrows, so as to provide the opportunity to point out the fact that they can point in opposite directions, but we prefer to normally keep the animation as simple and uncluttered as possible, and to use the definitional arrows only on an accompanying conventional schematic.

\section{Use of color}

The use of color is designed to make the images visually attractive and to convey additional information. As discussed in Section II, one way we use of color is for shading of wires to indicate potential. Black corresponds to ground and red corresponds to the maximum positive voltage encountered in the simulation; the intensity of the red varies smoothly between these two. This choice is based on the standard use of these two colors for wire color coding. For negative voltages, shading from black at ground to yellow at the largest magnitude negative voltage is used. Green is used for current arrows; although green is also standard as a color to indicate ground, we chose it for its association with motion, as in a traffic signal. Components use the remaining primary colors: blue, violet and orange. A copper-like shade of orange is used for inductor windings, corresponding to the material usually used, and helping to distinguish them from resistors and other passive components, in blue. Active components (e.g., sources and op-amps) are depicted in violet, which is perhaps a more "active" color than blue. The resulting colorful display is appealing as well as being functional.

\section{Implementation}

Animation of the visualization scheme described in Section II and shown in Figs. 1-5 has been implemented in a set of MATLAB functions we call the 3D Circuit Toolbox. An initial version of this toolbox may be downloaded freely from our web site ${ }^{11}$, and can be run with MATLAB versions 5.0 and above ${ }^{10}$ on a variety of platforms. This initial version of the software is simple, and lacks many capabilities that would be desirable. We share the hope expressed by Doering ${ }^{2}$ that automatic coupling to a circuit simulation program or even integration into a circuit simulation package will be eventually be realized. However, we also wished to make the process of implementing an animation of a new circuit as easy as possible in order to make use of these animations practical for ourselves and other educators pending availability of a more automated and flexible tool.

The 3D Circuit Toolbox ${ }^{11}$ comprises a set of MATLAB functions. To create an animation of a new circuit, one must write a new MATLAB script or function that consists mostly of calls to these functions. An example is included in the Appendix. A data structure for the circuit including graphical objects and other information is built up by a series of function calls, each of which adds a component or wire to the circuit. The circuit is constrained to a three-by-three grid of regularly spaced nodes. To add a component, one need only specify the numbers of the two nodes it is connected between, and the component type. This is similar to, but simpler than, the process of defining a circuit in a SPICE netlist. Similar function calls are used to add current arrows where they are desired. 
The three-by-three grid was initially chosen because it was believed that a larger circuit would be too complicated to follow in this type of visualization. However, there are a number of simple circuits that are difficult to fit on a three-by-three grid. For example, the animation of a buck switching power converter shown in Fig. 7 required placing the load resistor in an unusual position and adding a wire outside the normal three-by-three grid. Future versions will therefore include the capability of a larger grid. It may be that 3-D animations of more complex circuits will be valuable even though they will be difficult to follow, because the animation may still offer an easier way to gain insight on a complex circuit than the alternatives do.

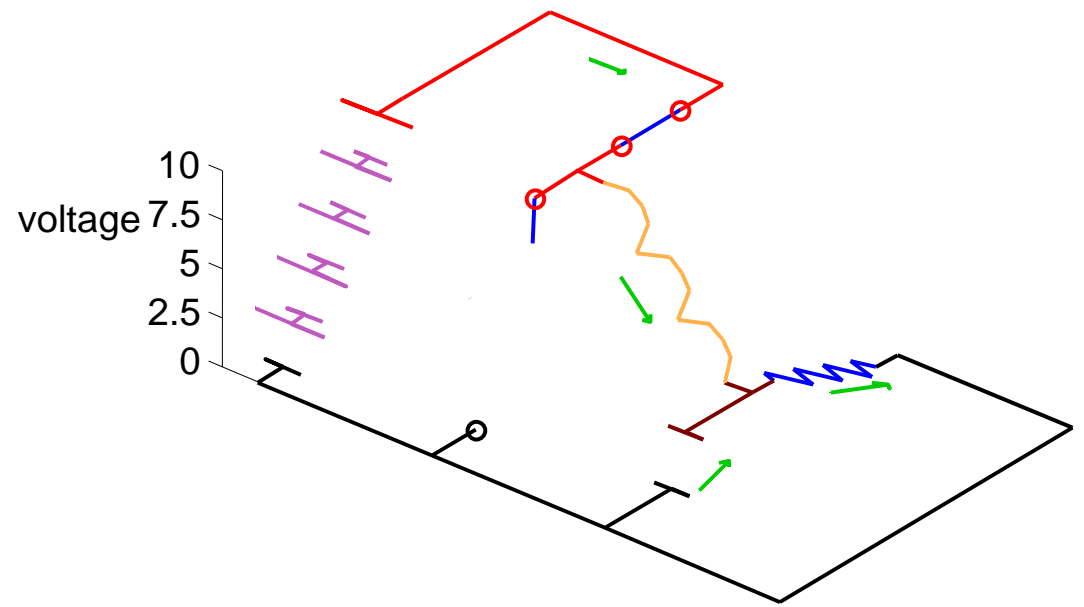

Fig. 7. Snapshot from an animation of a switching power converter (buck converter).

After defining the circuit and current arrows, one must also manually specify the dynamics of the circuit; no automatic calculation of dynamics is included. Often, one already knows simple equations for the behavior of a circuit one is presenting to a class, so a user who is familiar with MATLAB syntax can easily write a few lines of MATLAB code to calculate the circuit dynamics. What is needed is a matrix with the voltage at each of the nine nodes at the time corresponding to each frame in the animation, and a second matrix with the currents for each current arrow used. If switches, diodes, or other components that can visually change state are used, a matrix of their states must also be defined. The data for the circuit dynamics could also be generated by a simulation using MATLAB tools for solving ODEs, or by importing data from external circuit simulations.

After a call to an initialization routine, a call to an animation function produces an animation. Periodic functions can be implemented by putting this animation function call in a loop. The animation can be paused to wait for a keystroke at predetermined points, or the animation can be manually paused. The viewing angle of the three-dimensional scene can be set within the code defining the circuit, or can be manually changed with a mouse once the circuit is displayed.

In order to make it possible for students to easily view animations without installing MATLAB and the animation functions on their own computers, some animations have been converted to 
Graphical Interchange Format (GIF) using an inexpensive commercial tool ${ }^{12}$. This results in compact files compatible with web browsers. For the line art in these animations, GIF was found to be superior to video-oriented compression schemes, giving smaller file sizes and better quality. Examples can be seen at the 3D Circuit Toolbox web site ${ }^{11}$.

\section{Experience}

Still pictures and animations as described above have been used in the classroom and lab for a sophomore-level interdisciplinary lumped-element systems core course, and for an introductory electronics course. They will also be used in a power-electronics course, offered spring, 2002.

The systems course includes modeling and analysis of mechanical, electrical, thermal, fluid, chemical, and biological systems, and it is taken by all undergraduate engineering majors at Dartmouth regardless of their intended specialization. Students specializing in other areas often find the electrical content of the course particularly challenging, and this can be attributed in part to the difficulty of gaining intuition for voltage and current variables that cannot be directly observed. Other simultaneous improvements in the course made it difficult to directly assess the effect of the animation on students' attainment of learning objectives. However, the students' own assessment of its "effectiveness at helping [them] learn," as measured in a survey with 18 responses, was quite positive, as shown in Fig. 8. Six students wrote further comments, of which four were clearly positive (e.g., "Really helped me understand what was going on. Key!"), and the most negative was "Sometimes hard to understand/interpret."

The response of 19 students in the electronics course to a similar survey (also shown in Fig. 8) was even more positive, with more ratings of excellent and very good, three positive comments,

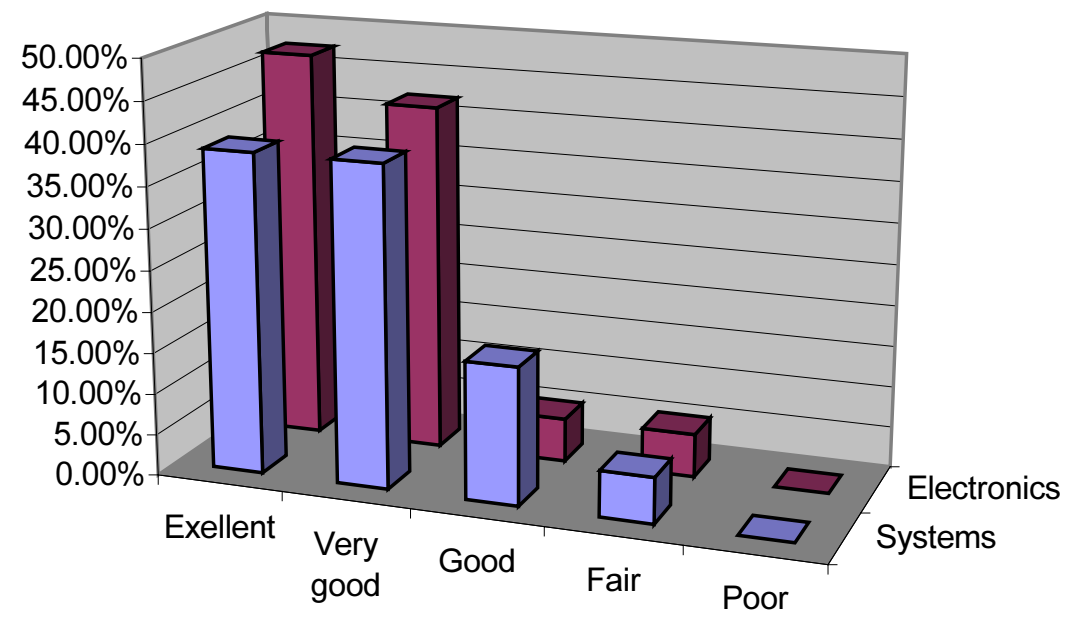

Fig. 8. Percentages of students giving the indicated ratings for the effectiveness of the 3D animations at helping them learn, in two different courses: interdisciplinary systems (front) and electronics (rear). 
and only one negative comment ("often hard to view correctly and understand what was going on"). The animations were the highest-rated of fifteen aspects of the course addressed in the survey.

A different type of circuit animation was also used in the systems course. This $2 \mathrm{D}$ animation was developed earlier, and used moving dots to represent current, varying densities of field lines to represent energy storage in a capacitor or inductor, and color to represent energy dissipation in a resistor. The average rating of this was lower by one-half point (between good and very good). Eight students rated the 3D animation higher, and only three rated the $2 \mathrm{D}$ simulation higher. An interesting observation is that the ratings of the $2 \mathrm{D}$ animation by those three were consistent with the class average; it was their ratings of the 3D animation that were different (and lower). This can be interpreted to indictate that the 3D animation does not work as well for all students, rather than indicating that the $2 \mathrm{D}$ animation was highly successful for some. Of the sixteen specific components of the course that students were asked to assess, only one, the set of written tutorial handouts, was rated significantly more effective in helping the students learn than the 3D circuit animations.

\section{Possible improvements}

Student reactions in class and in informal discussions indicate that it takes some time to understand the pictures, to see them in three dimensions, and to understand how the visualization corresponds to circuit variables. However, once this initial barrier has been overcome, most find it very useful. Since learning styles and three-dimensional visualization skills vary, some students may not ever get as much out of it as others. Improvements in the images might help to lower the initial barrier and to make the benefits available to more students.

More photorealistic images, with, for example, wires represented by cylinders rather than simple lines, and with simulation of lighting and shadows, could help make it easier to see the threedimensional aspect. A true stereoscopic visualization would probably be even more effective. The class could be outfitted with color-filter glasses, polarizing glasses, or shutter-glasses. For a large class, the first two of these are the most cost effective; for viewing outside of class, a small number of workstations could be outfitted with shutter-glasses.

It is not clear what the best way to visualize current really is. Thus, it might be valuable to include several options in animation system, so that the viewer can choose between them. This could allow selecting different ones for different situations when their particular advantages are needed, and could also facilitate testing the different methods of current visualization with the other aspects of the display kept constant in order to determine whether one method is more effective overall. Another option for current visualization would be to continue using arrows, but to also animate the thickness of the lines depicting wires and components according to the current magnitude. This would retain the advantages of the arrow system, while adding the advantage of the ribbon-height method ${ }^{2}$ in highlighting the primary current paths in a circuit. 
Of course, integration with a circuit simulation package, and improvements in the user interface are also highly desirable, to simplify the system's use for instructors, and more importantly, to make it accessible to students.

\section{Conclusion}

A 3D circuit animation system has been developed. Informal assessment indicates that it is highly effective in helping students learn. The animation follows previous visualizations in using elevation to represent voltage, and this is clearly a good choice, making Kirchoff's voltage law naturally and simply intuitive. The best choice to represent current is less clear, but arrows changing length according to current magnitude offer some advantages. A set of MATLAB functions makes it reasonably easy for an instructor to create an animation of a new circuit.

References

1. E.R. Doering, "Scientific visualization in the circuits curriculum: enhancing student insight." Proceedings of the Frontiers in Education 1995 25th Annual Conference, p. 2c6.13, 1995.

2. E. R. Doering, "CircuitViz: a new method for visualizing the dynamic behavior of electric circuits," IEEE Trans. Educ. 39, p. 297, 1996. Supplemental materials for this paper including animations are available at http://www.ece.msstate.edu/ hagler/Aug1996/005/cd/index.htm

3. A. del Rio and D. Valdes. “Three-dimensional model for analog circuit instruction,” IEEE Trans. Educ.,40, 1997.

4. U. Drofenik, J.W. Kolar, P.J. van Duijsen, P. Bauer. "New web-based interactive e-learning in power electronics and electrical machines." Conference Record of the 2001Industry Applications Conference, Thirty-Sixth IAS Annual Meeting. pp. $1858-1865$, vol.3.

5. D. Millard,W. Jennings, A. Sanderson, A. Wong, A. Patel, W. Brubaker, M. Perala, and D. Slattery. "Interactive learning modules for electrical, computer and systems engineering." Proceedings of the Frontiers in Education Conference, 27th Annual Conference. Teaching and Learning in an Era of Change, pp. 1165 -1170, vol.3, 1997.

6. C.L. Croskey, "A graphical animation authoring system for in-class demonstration of circuit operation." Proceedings on the Frontiers in Education Conference, pp. $171-176$.

7. S. Wirt, "The simple ciricuit," "The parallel circuit, and "The series circuit." http://www.sciencejoywagon.com/physicszone/lesson/07electr/default.htm, Science Joy Wagon, Lodi NY, 1998.

8. Edison, Version 4, http://www.designwareinc.com/edison.htm, DesignWare Inc., Meriden, CT, USA.

9. Dick White and Roger Doering. Electrical Engineering Uncovered. Second Edition. Prentice Hall, 2001, with 3D illustrations credited to Jim Hauser.

10. MATLAB, by Mathworks, http://www.mathworks.com/products/matlab/, Natick MA, USA.

11. http://engineering.dartmouth.edu/other/3Dcircuits

12. GIF Construction Set Professional, by Alchemy Mind Works, http://alchemy-mindworks.com/gifcon.html, Beeton, Ontario.

CHARLES R. SULLIVAN received a B.S. from Princeton University in 1987 and a Ph.D. from the University of California, Berkeley in 1996. Between degrees, he designed electronic ballasts at Lutron Electronics. He is presently Assistant Professor of Engineering at Dartmouth. He specializes in power electronics and magnetics, and has received an NSF CAREER award and an IEEE Power Electronics Society Prize Paper Award. 


\section{Appendix}

Sample file to specify a circuit and run an animation using the 3D circuit toolbox

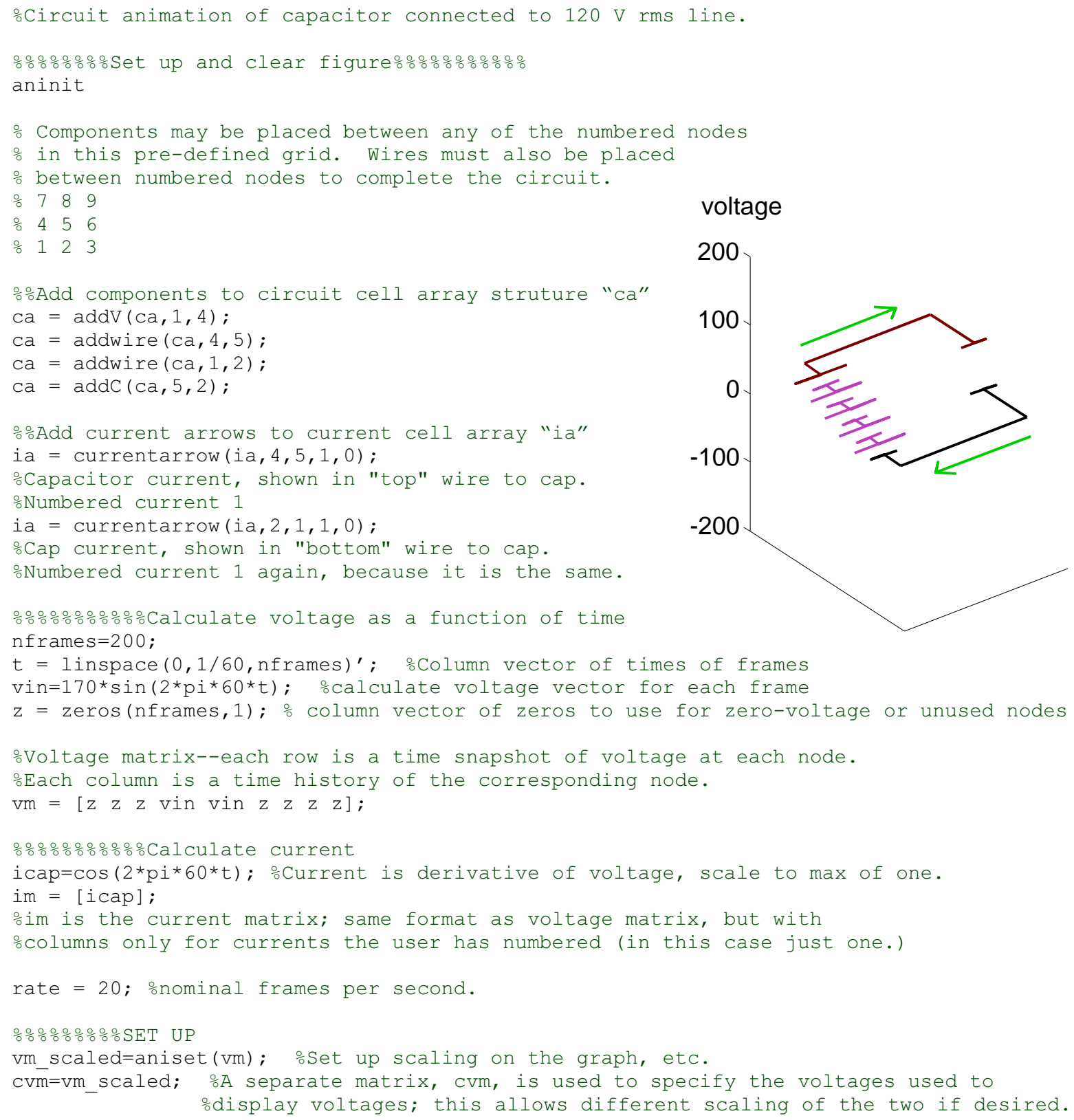

Proceedings of the 2002 American Society for Engineering Education Annual Conference \& Exposition Copyright (O) 2002, American Society for Engineering Education 